\title{
Vertical Retraction Syndrome: Clinical Features and Surgical Outcomes
}

\author{
Ebru Demet Aygit, Selcen Celik, Osman Bulut Ocak, Asli Inal, Ceren Gurez, Burcin Kepez Yildiz, \\ Korhan Fazil, Nilay Kandemir Besek, Birsen Gokyigit, Ahmet Demirok
}

University of Health Science Beyoglu Eye Training and Research Hospital, Istanbul, Turkey

\begin{abstract}
Objectives: The aim of this study was to report our clinical observations and the results of the surgical treatment of patients with vertical retraction syndrome (VRS).

Methods: Medical records were analyzed retrospectively and 5 patients with VRS were included in this study. A detailed ophthalmological examination and an orthoptic exam were performed. Superior rectus recession was performed in all cases and all of the patients were followed for at least 6 months.

Results: Analysis indicated that the mean patient age was 26.8 years. (min-max: 4-65 years). There were 3 females and 2 males in the group. Family history of a similar condition was positive in 2 patients. All of the patients had orthophoria following surgical treatment.

Conclusion: VRS is a rare, special form of retraction syndrome in which eye movement is limited by a fibrous band. Imaging was important to the surgical approach used in this group of patients to successfully treat the syndrome.

Keywords: Extraocular muscles, strabismus, vertical fibrosis syndrome.
\end{abstract}

\section{Introduction}

Congenital fibrosis of the extraocular muscles (CFEOM) has genetically defined strabismus syndrome subtypes (CFEOMIA, CFEOMIB, CFEOM2, CFEOM3A, CFEOM3B, CFEOM3C, Tukel syndrome, and CFEOM3 with polymicrogyria). These syndromes are characterized by congenital, non-progressive ophthalmoplegia, with or without ptosis, affecting part or all of the oculomotor nucleus and nerve (cranial nerve III) and muscles (superior, medial, and inferior recti, inferior oblique, and levator palpabrae superioris) innervated by this nerve. The trochlear nucleus, nerve (cranial nerve IV), and its innervated muscle (the superior oblique) may be involved in congenital fibrosis syndrome (CFS) (I). Recently, CFS was added to those classified as congenital cranial dysinnervation disorders (2). These syndromes are characterized by the replacement of normal tissue by fibrous tissue, which brings about a limitation of extraocular muscle movement. The clinical presentation is based on the number of affected muscles and the degree of fibrosis (3).

Vertical retraction syndrome (VRS) is a special form of CFS, and it is a rare kind of strabismus that consists of retraction of the globe with narrowing of the lid fissure in attempted elevation or depression. Frequently, elevation deficiency with variable retraction of the globe has been reported in the literature. In this study, the patients had depression deficiency and minimal retraction of the globe. The surgical goals were minimizing of head position, resolution of ptosis, and elimination or reduction of eye misalignment. Usually, correction of strabismus consisted of fibrotic muscle

Address for correspondence: Ebru Demet Aygit, MD. Department of Ophthalmology, Beyoglu Eye Training and Research Hospital, Istanbul, Turkey

Phone: +90 2122515900 E-mail: ebrudemet@hotmail.com

Submitted Date: September 19, 2017 Accepted Date: March 03, 2018 Available Online Date: April 03, 2018

${ }^{\circ}$ Copyright 2018 by Beyoglu Eye Training and Research Hospital - Available online at www.beyoglueye.com 
recessions, in some cases with ipsilateral muscle resection. The objective of this study was to report clinical observations and the results of surgical treatment of patients with VRS.

\section{Methods}

Medical records were analyzed retrospectively, and the following ophthalmological and orthoptic data were recorded: family history of similar conditions, age, sex, laterality, deviation, and restriction $(-5)-(0)$ of eye movement preoperatively and postoperatively. The study was performed in compliance with the principles of the Declaration of Helsinki.

The exclusion criteria of this study were a negative forced duction test (FDT), a history of orbital trauma, and other causes of ptosis: congenital or acquired ptosis, globe retraction with duction movements, myasthenia gravis, and different type of myopathy, and Marcus Gunn jaw-winking phenomenon.

A detailed ophthalmological examination, including a slit-lamp exam and fundoscopy, was performed on patients if possible, and visual acuity, deviation angle, and head turn were also examined. Visual acuity was assessed for each eye using the Snellen Chart. Cycloplegic refraction was performed after the administration of cyclopentolate eye drops. Patients had limited depression, and in 2 cases, a light retraction of the globe during downward gaze and eyelid lag. Deviation was measured using the Krimsky and the Hirschberg methods for near $(33 \mathrm{~cm})$ and distance $(6 \mathrm{~m})$ fixation.

A superior rectus (SR) muscle recession was preferred for depression deficiency. The degree of recession varied from 6 to $10 \mathrm{~mm}$, depending on the degree of hypertropia and the tightness of the muscle. Adhesions and fibrotic bands were released. If the treatment of horizontal strabismus was needed, the recession/resection procedures were performed taking the anterior segment circulation into account.

A forced duction test was performed on both eyes to identify restriction of vertical movement. A superior limbal conjunctival incision was used to access the SR muscle. At this point, the surrounding fascial attachments of the SR muscle were removed. Afterwards, a 6/0 vicryl (Ethicon, Inc., Somerville, NJ, USA) suture was used at each border of the muscle. Scleral reattachment of the SR muscle was performed according to the calculation of the recession. If the patient had a fibrotic band, it was noted. A topical steroid and antibiotic eye drops were used for the first postoperative week.

Examinations were performed on all patients on the postoperative first day, at the first week, first month, third month, and sixth month.

\section{Statistical Analysis}

The mean (SD) and frequency (percentage) were used to describe the summary data. The statistical analysis was performed using IBM SPSS Statistics for Windows, Version 20.0 (IBM Corp., Armonk, NY, USA). P $<0.05$ was considered statistically significant.

\section{Results}

The mean age at presentation was $30.6 \pm 23.4$ years (range: 4-65 years). The mean length of the follow-up period was 12 months (range: 6-24 months). There was a female dominance in our study, with a total of $3(60 \%)$ female patients. All of the patients were affected in the left eye. There was a positive family history of a similar condition in 2 patients. The mean preoperative hypertropia was $42 \pm 2.7$ PD, characterized by distinct, limited depression in the primary position (PP). After the third postoperative month, all of the patients displayed orthophoria. The primary surgery for all patients was recession of the SR muscle. Table I illustrates the demographic features of the study patients. Amblyopia was treated in 2 patients, but their visual acuity did not change.

Frequency doubling technology was performed at the start of the operation and all patients had positive results. Upon examination, the SR muscle in I patient was observed to be fibrotic and calcified. Two fibrotic tissues found in another patient were evaluated as muscle tissue. The SR muscle in the other 3 patients was tight and rigid.

Table I. Clinical characteristics of the 5 patients

\begin{tabular}{lcccccc} 
Patients & Age & Sex & Affected eye & $\begin{array}{c}\text { Preoperative } \\
\text { deviation }\end{array}$ & Surgery \\
\hline 1 & 14 & Male & Left & 45 & SRR & Orthophoria \\
2 & 4 & Female & Left & 40 & SRR & Orthophoria \\
3 & 35 & Female & Left & 40 & SRR & SRR \\
4 & 16 & Female & Left & 45 & Orthophoria \\
5 & 65 & Male & Left & 40 & SRR & Orthophoria \\
\hline
\end{tabular}




\section{Example Case}

A 14-year-old male presented with complaints of hypertropia from an early age in his left eye. An ophthalmological examination indicated that the right eye was normal and there was hypertropia in the left eye. The best corrected visual acuity was $10 / 10$ in the right eye and hand movement in the left eye. The anterior segment slit-lamp biomicroscopy was normal in the left eye, and the posterior segment was also normal. The decreased visual acuity in the left eye was attributed to deprivation amblyopia. A strabismus examination revealed 45 PD hypertropia and abduction, adduction, and depression deficiency in the left eye. The patient had not undergone any prior strabismus surgery. Recession of the SR muscle was planned. During the surgery, 2 fibrotic tissues were dissected from the area of the SR muscle. Following the operation, noticeable hypotropia in the PP was observed at the first week examination and 2 IU botulinum toxin $A$ (Botox; Allergan plc, Dublin, Ireland) was injected into the inferior rectus muscle. At the postoperative third month, orthophoria in the PP was recorded.

\section{Discussion}

Gast (4) reported a case of congenital external ophthalmoplegia in 1889 that was likely hereditary extraocular muscles fibrosis (4). In 1912, Bradbourne defined autosomal dominant extraocular muscle fibrosis with ptosis (5). Aebli first described congenital fibrosis of the extraocular muscle in 1933 (6). Brown et al. classified the following 5 types: (I) general fibrosis syndrome, (2) fibrosis of the inferior rectus with blepharoptosis, (3) strabismus fixus, (4) vertical retraction syndrome, and (5) unilateral fibrosis, blepharoptosis, and enophthalmos syndrome (7-9). Vertical retraction in 2 siblings has been well described by Khodadoust and Von Noorden (I0).

Congenital fibrosis of the extraocular muscles is characterized by the replacement of normal contractile muscle tissue with fibrous tissue or fibrous bands of varying degrees. Light and electron microscopy demonstrate the replacement of normal muscle with collagen and dense fibrous tissue with occasional areas of degenerated skeletal muscle. Cases may be unilateral or bilateral, and one or more than one muscle may be affected. Genetic factors may or may not have an impact (I I). Zhang et al. (I2) revealed that anomalous orbital structures may partially explain the cause of VRS and that it may also lead to atypical strabismus (I2).

Magnetic resonance imaging (MRI) can help to identify the presence of unusual eye movements. Yang et al. (I3) described the clinical features and MRI findings of 6 children with VRS in their study. They concluded that an anomalous orbital structure was the main cause of VRS and specific unusual eye movement. They added that MRI might assist in diagnosis. Zhang et al. (I2) presented the case of a girl with unilateral retraction in the upgaze and reported the MRI findings associated with retraction (I2). In our study, MRI did not indicate any pathology in the study patients.

The presence of an accessory extraocular muscle sometimes gives rise to restrictive strabismus. This only rarely appears in the literature (14). Molinari et al. (15) concluded that the presence of an accessory extraocular muscle should be included in the differential diagnosis of patients with atypical restrictive strabismus and especially when globe retraction is observed. Khitri and Demer (16) evaluated the orbital MRI of II 8 orthotropic and 453 strabismic patients, and 12 were noted to have these anomalous structures. In our study, we had I patient with an accessory fibrotic superior rectus muscle, which was dissected during surgery.

In these types of diseases, strabismus should be corrected before ptosis. Surgical correction has unpredictable outcomes in CFEOM because of the restrictive nature of the condition (17). The aim of surgical management is to achieve some functional readjustment of the ocular position and abnormal head posture.

Correction of vertical and horizontal strabismus was addressed with large muscle recession. Ptosis repair should aim at achieving a lid level I to $2 \mathrm{~mm}$ above the pupil in the PP to avoid exposure keratopathy. A rare condition, VRS may affect the SR or the inferior rectus muscle and result in depression or elevation of the eye. Therefore, surgery is performed on the muscle, and the area is examined for fibrotic tissue. In this study, orthophoria was obtained in all of the patients who had SR muscle recession surgery.

In the present study, all of the patients had left eye involvement. However, side dependence in VRS has not been reported in the literature.

There are some limitations to this study. First, we did not use imaging methods in all of the patients, and a genetic assessment was not possible. In addition, the number of patients studied was small, but this condition is very rare and specific. We believe that this study may be very useful to ophthalmologists who are interested in VRS.

Correction of vertical and horizontal strabismus is challenging in patients with congenital fibrosis of the extraocular muscles. To the best of our understanding, the surgical decision should be based on the clinical manifestations of the disease and the needs of the patient. Surgical recession of the affected muscle recession can be effective.

\section{Disclosures}

Acknowledgements: Ege Aygit contributed to the revision of the English-language spelling and grammar of this report.

Peer-review: Externally peer-reviewed.

Conflict of Interest: None declared. 
Authorship Contributions: Involved in design and conduct of the study (EDA, BG); preparation and review of the study (AD, EDA, SC, Al); data collection (EDA, OBO, CG, BKY, NKB); and statistical analysis (KF, EDA).

\section{References}

I. Whitman M, Hunter DG, Engle EC. Congenital Fibrosis of the Extraocular Muscles. In: Adam MP, Ardinger HH, Pagon RA, Wallace SE, Bean LJH, Stephens K, editors. GeneReviews. Seattle (WA): University of Washington; 1993-2018.

2. Singh A, Pandey PK, Agrawal A, Mittal SK, Rana KM, Bahuguna C. Congenital cranial dysinnervation disorders. Int Ophthalmol 2017;37:1369-8I. [CrossRef]

3. Harley RD, Rodrigues MM, Crawford JS. Congenital fibrosis of the extraocular muscles. J Pediatr Ophthalmol Strabismus 1978; I 5:346-58.

4. Peng JH, Huang FS, Liu Y, Chai HY, Li L, Gong SX, et al. A family history of congenital fibrosis of the extraocular muscle with autosomal dominant inheritance. Yi Chuan 2005;27:205-7.

5. Traboulsi El, Lee BA, Mousawi A, Khamis AR, Engle EC. Evidence of genetic heterogeneity in autosomal recessive congenital fibrosis of the extraocular muscles. Am J Ophthalmol 2000; 129:658-62. [CrossRef]

6. Hiatt RL, Halle AA. General fibrosis syndrome. Ann Ophthalmol 1983; I5: I 103-9.

7. Hertle RW, Katowitz JA, Young TL, Quinn GE, Farber MG. Congenital unilateral fibrosis, blepharoptosis, and enophthalmos syndrome. Ophthalmology 1992;99:347-55. [CrossRef]

8. Reck AC, Manners R, Hatchwell E. Phenotypic heterogeneity may occur in congenital fibrosis of the extraocular muscles. $\mathrm{Br}$ J Ophthalmol 1998;82:676-9. [CrossRef]

9. Brown H. Congenital structural muscle anomalies. In: Allen J, editor. Strabismus Ophthalmic Symposium. St. Louis: Mosby; 1950. p. 205-36.

10. Hansen E. Congenital general fibrosis of the extraocular muscles. Acta Ophthalmol (Copenh) 1968;46:469-76. [CrossRef]

II. Harley RD, Rodrigues MM, Crawford JS. Congenital fibrosis of the extraocular muscles. Trans Am Ophthalmol Soc 1978;76:197-226.

12. Zhang CY, Man FY, Wang ZC, Yu G, Wu Q, Jiao YH, et al. Magnetic resonance imaging of unilateral vertical retraction syndrome with atypical strabismus. Chin Med J (Engl) 20II;124:3195-7.

I3. Yang QI, Jiao YH, Man FY, Wang ZC, Chang QL, Lu W, et al. Vertical retraction syndrome caused by anomalous orbital structures. Zhonghua Yan Ke Za Zhi 20I I;47:983-8.

14. Lueder GT. Anomalous orbital structures resulting in unusual strabismus. Surv Ophthalmol 2002;47:27-35. [CrossRef]

15. Molinari A, Plager D, Merino P, Galan MM, Swaminathan M, Ramasuramanian S, et al. Accessory Extraocular Muscle as a Cause of Restrictive Strabismus. Strabismus 2016;24:178-83.

16. Khitri MR, Demer JL. Magnetic resonance imaging of tissues compatible with supernumerary extraocular muscles. Am J Ophthalmol 2010;150:925-31. [CrossRef]

17. Mohamad S, Jaafar MD, Traboulsi El. Clinical strabismus management, principles and surgical techniques. In: Rosenbaum AL, Santiago AP, editors. Congenital fibrosis of the extraocular muscles. Saunders Company; 1999. 